\title{
Efficacy of a combination of herbal gel versus topical diltiazem $(2 \%)$ in chronic anal fissure healing: a randomized double- blind clinical trial
}

\author{
Sedigheh Tavakoli-Dastjerdi ${ }^{1,2}{ }^{\mathbb{D}}$, Malihe Motavasselian ${ }^{3}$, Seyed Ahmad Emami ${ }^{4}$, Marjan Mansourian ${ }^{5}$, Amirhossein \\ Sahebkar ${ }^{6,7,8}$, Azam Teimouri ${ }^{9^{*} \mathbb{D}}$ \\ ${ }^{1}$ Department of Persian Medicine, School of Persian and Complementary Medicine, Mashhad University of Medical Sciences, Mashhad, Iran \\ ${ }^{2}$ Student Research Committee, School of Persian and Complementary Medicine, Mashhad University of Medical Sciences, Mashhad, Iran \\ ${ }^{3}$ Department of Persian Medicine, School of Persian and Complementary Medicine, Mashhad University of Medical Sciences, Mashhad, Iran \\ ${ }^{4}$ Department of Pharmacognosy, School of Pharmacy, Mashhad University of Medical Sciences, Mashhad, Iran \\ ${ }^{5}$ Department of Biostatistics and Epidemiology, School of Health, Isfahan University of Medical Sciences, Isfahan, Iran \\ ${ }^{6}$ Biotechnology Research Center, Pharmaceutical Technology Institute, Mashhad University of Medical Sciences, Mashhad, Iran \\ ${ }^{7}$ Neurogenic Inflammation Research Center, Mashhad University of Medical Sciences, Mashhad, Iran \\ ${ }^{8}$ School of Pharmacy, Mashhad University of Medical Sciences, Mashhad, Iran \\ ${ }^{9}$ Gastroenterology Department, Isfahan University of Medical Sciences, Isfahan, Iran
}

\section{A R T I C L E I N F O}

\section{Article Type:}

Original Article

\section{Article History:}

Received: 12 September 2018

Accepted: 24 January 2019

\section{Keywords:}

Anal fissure

Traditional medicine Allium ampeloprasum Astragalus sarcocolla Commiphora mukul Syzygium aromaticum Aloe vera

\begin{abstract}
A B S T R A C T
Introduction: Chronic anal fissure (CAF) is a common painful ailment that adversely affects quality of life. Nonsurgical treatments such as topical diltiazem are routinely administered to patients with CAF. However, diltiazem is associated with side effects such as headache and allergic reactions, which often lead to discontinuation of the treatment. The aim of this study was to determine the efficacy and safety of a topical Persian herbal remedy with analgesic, antiinflammatory and antiseptic properties versus topical diltiazem (2\%) in CAF healing.

Methods: In this randomized double-blind clinical trial, 70 patients with CAF were randomly divided into an intervention group receiving topical herbal medicine and control group receiving topical diltiazem (2\%) for 6 weeks. Both groups were given lifestyle and dietary advice including adherence to a high-fiber diet, avoidance of spicy foods, use of a laxative (psyllium powder) in case of constipation, and taking sitz baths. Severity of pain, bleeding, and itching were measured at the first visit as well as the first, third, and sixth weeks of treatment. Wound healing and epithelialization were evaluated by anal examination. The treatment and follow-up period was 12 weeks; 6 weeks for assessing the effect of treatment and 6 weeks for examining treatment maintenance and evaluating the percentage of recurrence.

Results: The herbal medicine caused significant reduction in pain compared with diltiazem $(P<0.001)$. The herbal medicine had a significant effect in terms of wound healing at the end of the period $(P<0.001)$. Recurrence rate was lower in the intervention group $(32 \%)$ than the control group (56\%), though this difference was not significant $(P=0.323)$

Conclusion: Findings of this clinical trial suggest a higher efficacy of the combination herbal remedy versus topical diltiazem $2 \%$ in the management of pain and wound associated with CAF.
\end{abstract}

Implication for health policy/practice/research/medical education:

The findings of this randomized controlled trial suggest the clinical efficacy of a topical herbal gel comprising Astragalus sarcocolla, Commiphora mukul, Allium ampeloprasum, Syzygium aromaticum and Aloe vera in the management of symptoms of chronic anal fissure.

Please cite this paper as: Tavakoli-Dastjerdi S, Motavasselian M, Emami SA, Mansourian M, Sahebkar A, Teimouri A. Efficacy of a combination of herbal gel versus topical diltiazem (2\%) in chronic anal fissure healing: a randomized double-blind clinical trial. J Herbmed Pharmacol. 2019;8(2):139-145. doi: 10.15171/jhp.2019.22.

\section{Introduction}

Anal fissure is a superficial rupture in the distal endoderm to the dentate line that usually occurs in the posterior coccygeal midline and sometimes in the anterior part. It is very prevalent such that $50 \%$ of people between 20 and 50 years old at least one time in their lifetime will 
suffer from an anal fissure (1). Furthermore, this is the main cause of acute pain of the anus and rectorrhagia in adults and children. More than $10 \%$ of patients referred to a proctology clinic suffered from this condition and this number is significantly increasing (2). Its lifetime incidence is $11.1 \%$ (3).

In chronic anal fissure (CAF), the symptoms usually last for more than 6 to 8 weeks. Its symptoms are severe acute pain during and after defecation, bleeding, and itching (4). The pain is described as sharp or cutting, and may last many hours after defecation. Acute pain scares the patients away from defecation and is associated with cyclical pain.

Pathogenesis of this disease, which is often described as "passing broken glass" is unknown; however, trauma occurs due to different causes such as constipation, hypertonicity of the internal anal sphincter (IAS), which leads to local ischemia and failure in restoration. Moreover, lifestyle, including diet and job conditions, affects the emergence and development of the disease (5).

The American Society of Colon and Rectal Surgeons (ASCRS) guideline advises non-surgery treatment including sitz baths, increased dietary fluid intake, laxatives, and topical nitrates (nitroglycerin), and topical calcium channel blockers like nifedipine and diltiazem. Side effects of these medications are headache in $20 \%$ $30 \%$ of patients, dizziness, faintness, and acute allergic reactions such as itching that hinder treatment. Moreover, recurrence is $50 \%$ after successful treatment with nitrates $(1,4)$. Gold standard treatment is surgery, including lateral internal sphincterotomy, which has side effects like fecal incontinence in one-third of patients after surgery, which may remain forever (6).

CAF decreases the quality of life of patients and acute chronic attacks are common (7). The anus is an outlet for body waste disposal through the digestive system, and if disease or functional disorder is lengthy in this region, its treatment will be difficult or impossible. Therefore, quick treatment has been advised (8).

Recently, treatment based on Local and Oral Antibiotics with Avoidance of Constipation (LOABAC) is advised (9). Thus, more research needs to be done on examining the effect of topical medications with analgesic, antiinflammatory and antimicrobial properties. On the other hand, treating constipation and preventing its recurrence requires dietary changes and correcting one's lifestyle. In complementary medicine and, in particular, Persian traditional medicine, which has a long history, offers holistic advice and recommendations for treating diseases and aliments such as anal fissures and gives special attention to diet and lifestyle. Therefore, in this study the effect of a topical medication that was a combination of extracts of Allium ampeloprasum (leek), Syzygium aromaticum (cloves) Commiphora mukul, Astragalus sarcocolla, and Aloe vera gel was examined on CAF considering disease recurrence or its chronic nature in Persian medicinal texts.

\section{Material and Methods}

Research design

This was a double-blinded random clinical trial conducted on 112 female patients with CAF from March 2017 to December 2017 who were referred to a gastroenterology clinic.

\section{Sampling}

The study population consisted of women with CAF who were referred to the gastroenterology clinic of Al-Zahra hospital from hospitals dependent on the Isfahan Medical Sciences University. Examination and diagnosis were done by a gastroenterologist. Colonoscopy was done on patients with rectorrhagia or suspected of having other colon diseases. Patients with an atypical anal fissure and/ or underlying disease like irritable bowel disorder or malignancies, and pregnant women were not included.

\section{Sample size}

Due to the lack of a similar study merely based on the opinion of experts, the sample size was calculated in two independent groups with $95 \%$ confidence and $80 \%$ power. The minimum sample size was considered 35 in each group. Five participants dropped out of the intervention; hence, 65 of the women were analyzed.

\section{Selection criteria}

CAF patients with or without rectorrhagia who were diagnosed by a gastroenterologist were included into the study, after their description of symptoms and history were recorded and a physical examination was done.

\section{Intervention}

Eating a high fiber diet, avoiding spicy foods, using laxatives in case of constipation, and taking sitz baths twice a day were advised to both groups. The laxative used in the study was psyllium powder produced by Iran Darouk Company. One gram, twice a day of diltiazem 2\% (Sobhan Daru Company) was given to the control group, around the anus and inter-anus. The intervention group received a gel that was a combination of $A$. sarcocolla, $C$. mukul, A. ampeloprasum (leek), S. aromaticum (cloves) and $A$. vera gel as 1 gram twice a day, around the anus and inter-anus. Treatment and follow-up period was 12 weeks: 6 weeks for assessing the effect of the treatment and 6 weeks for examining treatment maintenance and percentage of recurrence.

Outcome and data collection tool

\section{Outcome}

At first, the measured outcomes (pain, bleeding, and itching) of the patients were examined by the Persian 
questionnaire and percentage of wound healing and recurrence was checked by anal examination. One part of the questionnaire included demographic characteristics of the patient, duration of the disease, and accompanying diseases, and the other part included the visual analogue scale (VAS), which is a ten $\mathrm{cm}$ linear tool between 0 and 10 that uses a table to measure pain, bleeding, and itching (zero $=$ no pain or bleeding or pruritus, $1-3=$ mild, $4-6=$ moderate, $7-9=$ severe, and very severe $=10$ )

Allocation, blinding, and randomization

Patients were divided into intervention and control groups using a computer random-numbers table and allocation. Then, similar packages of the medication were prepared, one with the control medication and the other including the intervention medication. The patients and physician were blind to the kind of treatment of each group. Therefore, this study was randomized and double-blind.

\section{Research ethics}

This study was approved by the MUMS ethical committee (No. IR. MUMS.REC.1395.483). Patients received necessary information on the study and signed a consent form in Persian. Patients' inclusion into the study was optional and they were allowed to withdraw from the study whenever they wanted. Lastly, all the participants' data were confidential. Contact numbers were provided to patients if they had any queries or needed medical help.

Preparation of the herbal medicine

First, hydroalcoholic extracts of A. ampeloprasum (5 units), A. sarcocolla (1 unit), C. mukul (1 unit), and S. aromaticum
(0.5 unit) were prepared by a pharmacognosist, and then the expert made the obtained extract into $30 \mathrm{~g}$ tubes by combing $A$. vera gel as $20 \%$ gel in a sodium carboxymethyl cellulose gel base. Plants were from the Mashhad medical plants market. Amounts and ratios of the plants in this compound were determined based on the references of Persian medicine and recommendations of the pharmacognosist.

\section{Statistical methods}

Analysis was done in the descriptive and analytical parts. In the descriptive part, characteristics of the studied subjects were presented, generally and by group, through providing number (percentage) for qualitative variables, and mean and standard deviation for quantitative variables. Comparison between the two groups was done based on the different variables using the chi-square test for qualitative variables and the independent $t$ test for quantitative variables. Also, when the normal distribution assumption was not met for quantitative variables, the Mann-Whitney test was used. In the analysis, generalization estimation equations (GEE) were used for comparison between the two groups, response variables such as pain, bleeding and so on were not quantitative, and the study type was followed-up during treatment. All analyses were done at $0.05 \%$ error level using SPSS20 software.

\section{Results}

Of 112 patients, 70 patients who met the inclusion criteria were included into the study as shown in Figure 1. These patients were divided into two groups: control group (35

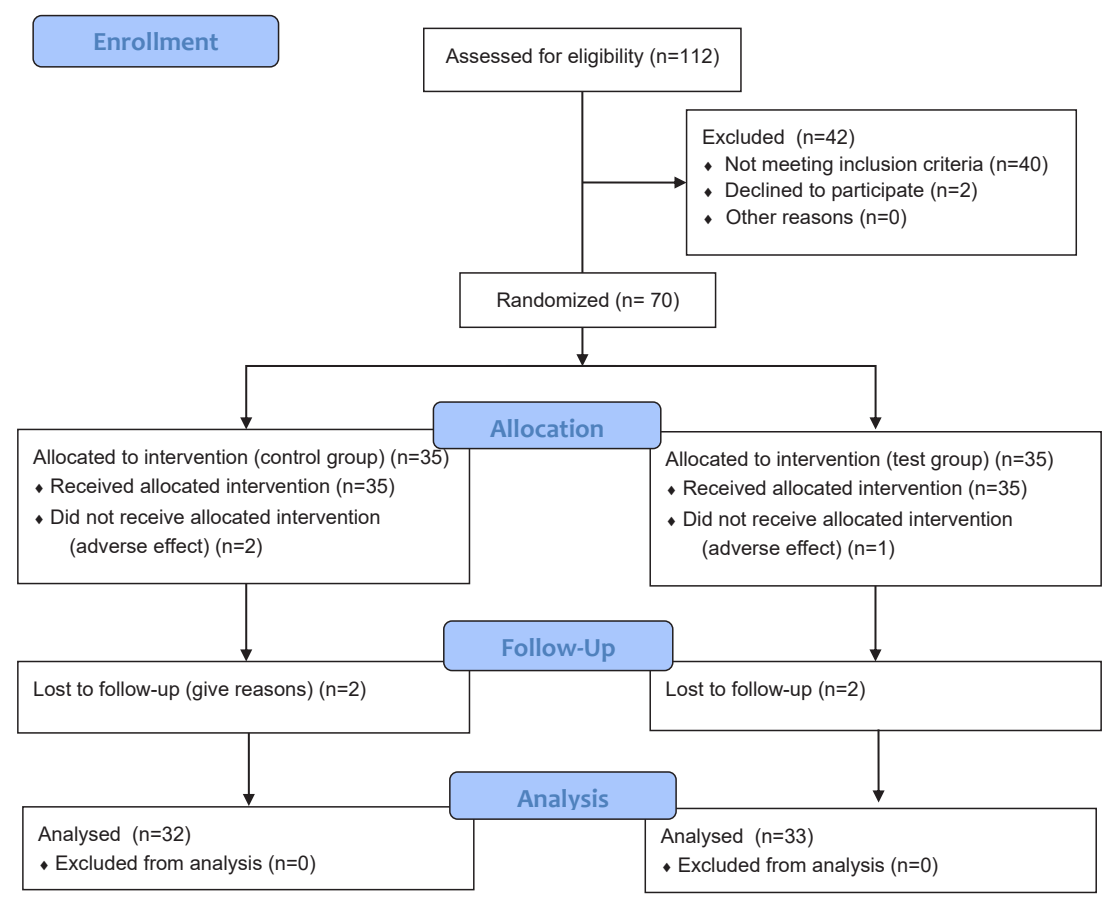

Figure 1. Consort flowchart of the study. 
patients) and intervention group (35 patients) with mean age of $39.61 \pm 10.09$. The patients' mean age was different between the two groups $(P=0.02)$ and this variable was mediated by the Mann-Whitney test. Duration of the disease, which was a confused variable in both groups, did not have normal distribution between the two groups $(P<0.001)$ and was mediated (Table 1$)$. In the control group, rectorrhagia at the time of referral was reported in 10 patients $(31 \%)$ and in the intervention group in 18 patients $(52.9 \%)$. In the control group, itching was reported in 14 patients $(43.8 \%)$ and in the intervention group in 11 patients $(32.4 \%)$. The control group received diltiazem as treatment medication and the intervention group received the desired herbal medicine. In the control group, two patients discontinued the treatment because of experiencing headaches and one patient for lack of recovery. In the intervention group, one patient exited the study for experiencing severe itching and allergy to the medication and one patient for becoming pregnant (Figure 2). Mean pain score at the follow-up times during the treatment period (first visit, weeks 1, 3, and 6) was recorded in Table 2. At the time of referring, the pain score did not have a significant difference between the two groups, and herbal medicine had a good effect in terms of decreasing pain $(P<0.001)$ (Table 2$)$. Although changes in the frequency of bleeding and itching were not significant (Tables 3 and 4), the herbal medicine had a significant effect in terms of wound healing at the end of the period $(P<0.001)$. Percentage of recurrence was lower in the intervention group than the control group, although there was no verifiable impact on recurrence $32 \%$ ( in the

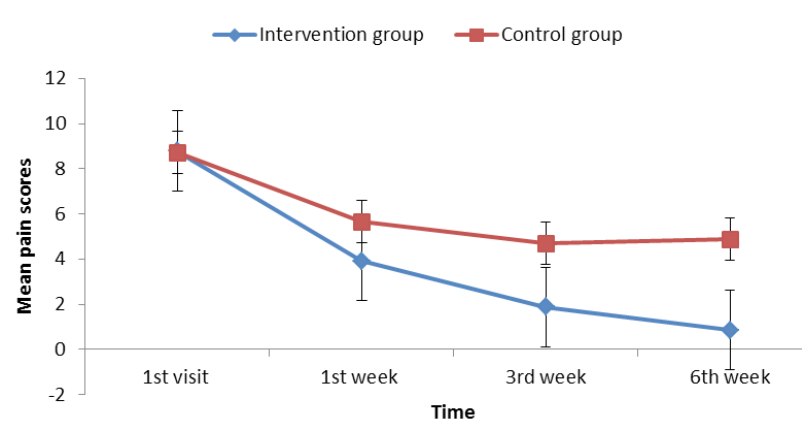

Figure 2. Pain score during the study period.

intervention group and 56\% in the control group).

\section{Discussion}

Based on the obtained results, the decrease in patients' pain scores and percentage of wound healing was significant in the intervention group (Table 2). There was no significant difference between the two groups in terms of bleeding and itching (Tables 3 and 4). There was also no significant difference between the two groups in terms of recurrence.

In some studies, decrease in the pain scores and in other studies wound healing has been considered as recovery. In fact, because an anal fissure has a particular and different appearance, wound healing is a good criterion for measuring recovery (10). Epithelialization means wound healing and lack of ulcer at the wound site. In CAF, IAS spasms cause pain, and decreasing pressure and spasms in this area during treatment will decrease the pain of patients. Despite having only an acute anal fissure, wound healing is not possible without medicine (due to internal

Table 1. Demographic data of participants

\begin{tabular}{|c|c|c|c|c|}
\hline Characteristics & Intervention & Control & Total & $P$ value \\
\hline \multicolumn{5}{|l|}{ Education level, No. (\%) } \\
\hline No diploma & $28(82.4)$ & $27(84.4)$ & $55(83.3)$ & \multirow{2}{*}{0.392} \\
\hline Diploma and higher & $6(17.6)$ & $5(15.6)$ & $11(16.7)$ & \\
\hline \multicolumn{5}{|l|}{ Marital status, No. (\%) } \\
\hline Single & $4(11.8)$ & $4(12.5)$ & $8(12.1)$ & \multirow{2}{*}{0.502} \\
\hline Married & $30(88.2)$ & $28(87.5)$ & $58(87.9)$ & \\
\hline \multicolumn{5}{|l|}{ Employment, No. (\%) } \\
\hline Housewife & $27(79.4)$ & 23 (71.9) & $50(75.8)$ & \multirow{3}{*}{0.336} \\
\hline Employed & $6(17.6)$ & $8(25)$ & $14(21.2)$ & \\
\hline Student & $1(2.9)$ & $1(3.1)$ & $2(3)$ & \\
\hline Age (y) & $38.21+9.17$ & $41.09+10.82$ & $39.61+10.09$ & 0.02 \\
\hline Duration of disease (mon) & $12+(45-12)$ & $36(102-12)$ & $18(48-12)$ & 0.001 \\
\hline Ant fissure, No. (\%) & $9(26.5)$ & $6(18.8)$ & $15(22.7)$ & 0.88 \\
\hline Post fissure, No. (\%) & $30(88.2)$ & $30(93.8)$ & 60 (90.9) & 0.089 \\
\hline Skin tag, No. (\%) & $16(47.1)$ & $15(46.9)$ & $31(47)$ & 0.537 \\
\hline
\end{tabular}

Table 2. Mean pain scores during the treatment period (first visit, weeks 1,3 , and 6 )

\begin{tabular}{llllr}
\hline Time & 1st visit & 1st week & 3rd week & 6th week \\
\hline Intervention group & $8.79 \pm 1.95$ & $3.91 \pm 2.54$ & $1.88 \pm 2.07$ & $0.85 \pm 1.27$ \\
Control group & $8.72 \pm 2.41$ & $5.66 \pm 2.88$ & $4.70 \pm 2.98$ & $4.87 \pm 4.05$ \\
\hline
\end{tabular}


Table 3. The healing of hemorrhage in groups

\begin{tabular}{|c|c|c|c|c|c|c|}
\hline Time & Bleeding & 1st visit & 1st week & 3rd week & 6th week & $P$ value \\
\hline \multirow{2}{*}{ Intervention group } & Yes & 18 (52.9) & $3(8.8)$ & $1(2.9)$ & $2(5.9)$ & \multirow{4}{*}{0.11} \\
\hline & No & $16(47.1)$ & $30(88.2)$ & $32(94.1)$ & $31(91.2)$ & \\
\hline \multirow{2}{*}{ Control group } & Yes & $10(31.3)$ & $8(25)$ & $9(28.1)$ & $8(25)$ & \\
\hline & No & $22(68.8)$ & $24(75)$ & $21(65.6)$ & 23 (71.9) & \\
\hline
\end{tabular}

Table 4. The healing of itching in groups

\begin{tabular}{|c|c|c|c|c|c|c|}
\hline Time & Itching & 1st visit & 1st week & 3rd visit & 6th visit & $P$ value \\
\hline \multirow{2}{*}{ Intervention group } & Yes & $11(32.4)$ & $9(26.5)$ & $8(23.5)$ & $2(5.9)$ & \multirow{4}{*}{0.214} \\
\hline & No & $23(67.6)$ & $24(70.6)$ & $25(73.5)$ & $30(88.2)$ & \\
\hline \multirow{2}{*}{ Control group } & Yes & $14(43.8)$ & $11(34.4)$ & $9(28.1)$ & $8(25)$ & \\
\hline & No & $18(56.3)$ & $21(65.6)$ & $21(65.6)$ & 23 (71.9) & \\
\hline
\end{tabular}

sphincter spasms and fibrosis). In different studies, about $50 \%$ of CAF patients have had epithelialization after 6 weeks of treatment $(5,10)$.

Effects of nitrates and calcium channel blockers in CAF treatment have been reported between $40 \%$ and $69 \%$ (10). Based on the Association of Coloproctology of Great Britain and Ireland (ACPGBI), the most successful treatment of CAF has been reported with topical diltiazem. Based on the ACPGBI algorithm, CAF treatment includes constipation relief and topical diltiazem, in which successful treatment and wound healing were reported at 83.3\% using this algorithm (3). In another study, wound healing with oral diltiazem and topical diltiazem was reported at $40 \%$ and $80 \%$, respectively (11). In our study, wound healing was about $87 \%$ in the intervention group and $43 \%$ in the control (diltiazem) group. Of course, mean duration of the disease and mean age of patients were more in the control group, which probably affected the result of treatment.

Recurrence was examined 6 weeks after the end of treatment. In this study, recurrence was $56 \%$ in the control group and $32 \%$ in the intervention group. Regarding the wax and wane mode of this disease, recurrence is prevalent (12). In different studies, about 50\% recurrence has been reported. In a study with long-term follow-up (1 year), patients had high recurrence $(67 \%)$ despite the high rate of epithelialization, and it was stated that the effect of treatment with GTN in terms of preventing CAF recurrence was frustrating (13). A high fiber diet, avoiding spicy foods, and paying attention to health in the rectal region are important in preventing recurrence (14).

In Persian medicine, removing the cause of disease is important and in CAF, constipation and lifestyle are leading causes of this ailment. Therefore, avoiding spicy foods, having a high fiber diet, and controlling stress have been advised. From the classical medicine viewpoint, IAS physiology is regulated by the intestine and autonomic neural system, and sympathetic system has a close relationship with stress. Consequently, stress and psychological problems can intensify sympathetic function disorder and provide conditions for or further develop of CAF (15). Using a sitz bath to decrease spasms and pain, and using more effective medicines has been advised in Persian and classical medicine (16). Also, using psyllium (Plantago ovata) as a laxative has been advised in Persian and classical medicine because it increases feces volume $(7,17,18)$. Therefore, percentage of recurrence in CAF decreases by observing recommendations based on Persian medicine. Studies have reported that epithelization chance in CAF is very low when the duration of the disease is more than 6 months (10).

Regarding the LOABAC theory, using topical medicine with anti-inflammatory, antimicrobial, and sedative effects is needed in CAF treatment (9). In Persian medicine, $A$. vera, A. ampeloprasum (leek), and C. mukul have been considered useful in anus diseases (16). In addition, Astragalus sarcocolla has been advised for wound healing $(8,19,20)$.

Various studies have proven the antimicrobial, antioxidant, anti-inflammatory, and anti-cancer effects of $S$. aromaticum. Anti-inflammatory effect of the alcoholic extract of oral S. aromaticum has been proven in animal studies without reported side effects (21). In a study, it was found that clove oil cream was effective in wound healing and decreasing sphincter pressure of the anus (22). A. vera gel has been reported effective in treating wounds, skin fungal infections, common cold, and constipation (23). The effect of $A$. vera gel (Aloe vera barbadensis) on CAF was studied and it was revealed that it was effective in wound healing (24). Aloe vera barbadensis heals wounds due to its astringent effect and it does not cause irritation due to its anti-inflammatory effect (24-26). C. mukul has anti-inflammatory, expectorant, antiseptic, astringent, and demulcent properties (27). The most 
important phytochemicals of leek are flavonoids and saponins. Flavonoids have a positive effect on hemorrhoid and saponins have anti-steroid, anti-inflammatory, and epithelialization effects. In examining the effect of leek extract cream on hemorrhoid, it was found to be effective in controlling bleeding and decreasing pain and irritation (28). Anti-fungal effects of leek have also been proven (29). In addition, fresh leek is a good source of nitrates (30) and it may be effective on CAF treatment. With regard to the effects of all mentioned plants and their synergetic effects when combined, our herbal medicine was effective in decreasing pain, wound healing, and recurrence as was predicted.

It was not possible to have a placebo group because of ethical issues, since study subjects would have been subjected to pain and discomfort. This is one of the limitations of the study. In terms of recurrence follow-up, if patients were supervised for a longer time, the effect of the medicine could have been evaluated better. In terms of duration of disease, and regarding the reverse relationship between duration of disease and recovery, if patients with disease duration lower than 6 months were included in the study, they would have been more confident in terms of response to treatment.

\section{Conclusion}

Findings show that herbal medicine with the desired ingredients can be effective in CAF treatment and increase quality of life due to their effect on pain relief and reduced recurrence.

\section{Authors' contributions}

STD wrote the manuscript; STD, MM and AT performed the research; MM and SAE designed the research study; MM, AT and AS coauthored and critically revised the manuscript; MM analyzed the data. All authors approved the final version of the manuscript

\section{Conflicts of Interest}

The authors declare there is no conflict of interest in the study.

\section{Ethical considerations}

Ethical issues (including plagiarism, misconduct, data fabrication, falsification, double publication or submission redundancy) have been completely observed by the authors. The protocol was confirmed through Ethical Committee of Mashhad University (Code no: IR.MUMS. REC.1395.483). The trial was registered with the Iranian Registry of Clinical Trials (http://www.irct.ir; Identifier: IRCT2017010731808N1).

\section{Funding/Support}

This research was financially supported by the Research Council at the Mashhad University of Medical Sciences,
Mashhad, Iran. (grant No. 950357).

\section{References}

1. Salari M, Salari R, Dadgarmoghadam M, Khadem-Rezaiyan M, Hosseini M. Efficacy of egg yolk and nitroglycerin ointment as treatments for acute anal fissures: A randomized clinical trial study. Electron Physician. 2016;8(10):3035-41. doi: $10.19082 / 3035$.

2. Sudesh G, Bala M, Bhawana G, Manpreet S. Indicating the finest treatment for chronic fissure in ano. International Journal of Ayurveda and Pharma Research. 2017;5(11):438.

3. Farkas N, Solanki K, Frampton AE, Black J, Gupta A, West NJ. Are we following an algorithm for managing chronic anal fissure? A completed audit cycle. Ann Med Surg (Lond). 2016;5:38-44. doi: 10.1016/j.amsu.2015.11.008.

4. Sahebally S, Meshkat B, Walsh S, Beddy D. Botulinum toxin injection versus topical nitrates for chronic anal fissure- an updated meta-analysis of randomised controlled trials. Gut. 2017;66(Suppl 2):A137-98. doi: 10.1136/ gutjnl-2017-314472.270.

5. Anal fissure - Symptoms, diagnosis and treatment. BMJ Best Practice website. Available from: http://bestpractice. bmj.com/topics/en-us/563. Accessed February 28, 2018.

6. Madalinski MH. Identifying the best therapy for chronic anal fissure. World J Gastrointest Pharmacol Ther. 2011;2(2):9-16. doi: 10.4292/wjgpt.v2.i2.9.

7. Poh A, Tan KY, Seow-Choen F. Innovations in chronic anal fissure treatment: A systematic review. World J Gastrointest Surg. 2010;2(7):231-41. doi: 10.4240/wjgs.v2.i7.231.

8. Avicenna. The Canon of medicine. Translated by Sharafkandi AR. Tehran: Soroush Press; 2008. [Persian].

9. Garg P, Lakhtaria P, Gupta V. Oral plus local antibiotics significantly reduce the need for operative intervention in chronic anal fissure: a novel finding. Indian J Surg. 2018;80(5):415-20. doi: 10.1007/s12262-017-1617-z.

10. Emile SH, Elgendy H, Elfeki H, Magdy A, Abdelmawla AA, Abdelnaby M, et al. Does the duration of symptoms of anal fissure impact its response to conservative treatment? A prospective cohort study. Int J Surg. 2017;44:64-70. doi: 10.1016/j.ijsu.2017.06.044.

11. Richa K, Ashwani K, Priyanka B, Vineeta S, Doctor SR. A prospective randoized comparative evaluation of oral diltiazem and topical diltiazem (2\%) ointment in the treatment of chronic anal fissure. World J Pharm Pharm Sci. 2017;6(10):1489-99.

12. Nelson RL, Thomas K, Morgan J, Jones A. Non surgical therapy for anal fissure. Cochrane Database Syst Rev. 2012(2):Cd003431. doi: 10.1002/14651858.CD003431. pub3.

13. Nelson RL, Manuel D, Gumienny C, Spencer B, Patel K, Schmitt $\mathrm{K}$, et al. A systematic review and meta-analysis of the treatment of anal fissure. Tech Coloproctol. 2017;21(8):605-25. doi: 10.1007/s10151-017-1664-2.

14. Gupta PJ. Family practice in ano-rectal diseases. Pak J Med Sci. 2006;22(1):82-7.

15. Arisoy O, Sengul N, Cakir A. Stress and psychopathology and its impact on quality of life in chronic anal fissure (CAF) patients. Int J Colorectal Dis. 2017;32(6):921-4. doi: 10.1007/s00384-016-2732-1. 
16. Derakhshan AR. Natural Treatments for Fissure in Ano Used by Traditional Persian Scholars, Razi (Rhazes) and Ibn Sina (Avicenna). J Evid Based Complementary Altern Med. 2017;22(2):324-33. doi: 10.1177/2156587216650302.

17. Nimrouzi M, Zarshenas MM. Holistic approach to functional constipation: Perspective of traditional Persian medicine. Chin J Integr Med. 2015. doi: 10.1007/s11655015-2302-3.

18. Mozaffarpur SA, Naseri M, Esmaeili Dooki MR, Bijani A, Kamalinejad M, Yousefi M, et al. Introduction of natural medicinal materia effective in treatment of constipation in Persian traditional medicine. Hist Med J. 2012;3(9):79-95.

19. Hooper D, Field H. Useful plants and drugs of Iran and Iraq. Chicago: Biodiversity Heritage Library; 1937. doi: 10.5962/bhl.title.2281.

20. Ghahramani Dehbokri S, Saeidiani S, Mohammadzadeh R, Shoja Gharabagh M, Asalforoush Rezaeieh A, Akradi L. A comparative study of the healing effects of calendula and Astragalus fasciculifolius aqueous resin extract on rabbit skin wounds. J Vet Med. 2010;3(9):51-61.

21. Saeed TA, Osman OA, Amin AE, El Badwi SMA. Safety assessment and potential anti-inflammatory effect of ethanolic extract of Syzygium aromaticum in albino rats. Adv Biosci Biotechnol. 2017;8(11):411-20. doi: 10.4236/ abb.2017.811030.

22. Elwakeel HA, Moneim HA, Farid M, Gohar AA. Clove oil cream: a new effective treatment for chronic anal fissure. Colorectal Dis. 2007;9(6):549-52. doi: 10.1111/j.14631318.2006.01185.x

23. Kumar S, Pande SK, Yadav JK, Saini R, Kumar S, Yadav SK, et al. Effect of fungicides and botanicals against leaf rot disease caused by Fusarium oxysporum in Aloe vera
(Aloe barbadensis Miller). Int J Curr Microbiol Appl Sci. 2017;6(10):2957-62. doi: 10.20546/ijcmas.2017.610.349.

24. Ansari MS, Nizam R, Ahmad W. Local application of Safoof-e-aelwa in fissure in Ano: A case series. Int J Med Res. 2017;2(6):31-3.

25. Rahmani N, Khademloo M, Vosoughi K, Assadpour S. Effects of Aloe vera cream on chronic anal fissure pain, wound healing and hemorrhaging upon defection: a prospective double blind clinical trial. Eur Rev Med Pharmacol Sci. 2014;18(7):1078-84.

26. Gautam S, Verma K. Effect of Aloe vera and mulethi with honey in the management of fissure in ano. World J Pharm Med Res. 2017;3(8):411-4.

27. Yousefi M, Hoseini SM, Salari R. Evaluating the clinical efficacy of Guggulu resin on constipation: A randomised clinical trial. Adv Integr Med. 2016;3(3):98-102. doi: 10.1016/j.aimed.2016.11.004.

28. Mosavat SH, Ghahramani L, Sobhani Z, Haghighi ER, Heydari M. Topical Allium ampeloprasum subsp iranicum (Leek) extract cream in patients with symptomatic hemorrhoids: a pilot randomized and controlled clinical trial. J Evid Based Complementary Altern Med. 2015;20(2):132-6. doi: 10.1177/2156587214567954.

29. Sadeghi M, Zolfaghari B, Senatore M, Lanzotti V. antifungal cinnamic acid derivatives from Persian leek (Allium ampeloprasum subsp. persicum). Phytochem Lett. 2013;6(3):360-3. doi: 10.1016/j.phytol.2013.04.007.

30. Zaki EF, Khallaf MF. Effect of adding leek as a natural source of nitrite on the processing and quality properties of camel sausage during frozen storage. J Adv Food Sci Technol. 2018;5(1):27-33. 